\title{
Importancia de la armada en el tráfico ilegal de especies marinas
}

\author{
Mario Alberto Correa Segura ${ }^{1}$ \\ David Alberto Vela Ordoñez ${ }^{2}$
}

\section{Resumen}

El tráfico ilegal de especies marinas es una problemática ambiental, social, cultural y económica que afecta a la sociedad Colombia, es un atentado contra biodiversidad del país que pone en riesgo múltiples ecosistemas marinos, generando un desequilibrio ambiental que trae serias repercusiones para la flora, fauna y la especie humana en general. Es por esto que la Armada Nacional cumple una labor fundamental en el ejercicio indispensable en la intervención y detención del tráfico ilegal de especies marinas, con el apoyo de otras instituciones ambientales, gubernamentales y hacedoras del bienestar y orden social como la policía y la rama judicial para la protección de ecosistemas de zonas marítimas en el Pacífico y el Caribe, fluviales y algunas áreas terrestres que incluyen los puertos de acuerdo a lo establecido por el Ordenamiento Marino Costero. Dicho esto, el objetivo del presente estudio es analizar el rol de la Marina Colombiana como institución empoderada para la protección de los recursos marinos renovables y no renovables.

\section{Palabras Claves}

Biodiversidad, Tráfico ilegal, Especies marinas, Medio Ambiente, Armada Nacional.

\begin{abstract}
The illegal traffic of marine species is an environmental, social, cultural and economic problem that affects the Colombian society, it is an attack against the country's biodiversity that threatens multiple marine ecosystems, generating an environmental imbalance that brings serious repercussions for the flora, fauna and the human species in general. This is why the Navy is a valuable institution that appropriates the protection of renewable and non-renewable marine resources, therefore its exercise in the intervention and detention of illegal trade in marine species is essential, as is the support of other environmental institutions, governmental and welfare makers and social order such as the police and the judicial branch for the protection of ecosystems of maritime zones in the Pacific and the Caribbean, fluvial and some terrestrial areas that are connected in the area of special protection in seas and ports of agreement to what is established by the Coastal Marine Ordinance. This said, the objective of this study is to analyze the roll of the Colombian Navy as an empowered institution for the protection of renewable and nonrenewable marine resources.
\end{abstract}

\section{Keywords}

Biodiversity, Illegal trafficking, Marine species, Environment, National Navy.

1. Estudiante Curso Básico, Facultad de Ciencias Navales y Náuticas, Escuela Naval de Cadetes “Almirante Padilla”, Mario.correa.se@armada.mil.co. 2. Estudiante de curso Básico, Facultad de Ciencias Navales y Náuticas, Escuela Naval de Cadetes “Almirante Padilla”, Diego.vela@armada.mil.co. 


\section{Introducción}

Las especies marinas establecen un grupo taxonómico muy amplio y variado que enriquece la biodiversidad de Colombia que fortalece el buen estado ambiental de los ecosistemas, sin embargo en los últimos años están sufriendo una alta presión por las diferentes actividades humanas como la contaminación, la eliminación de zonas ecológicas por la apropiación urbana e industrial y el tráfico ilegal de muchas especies como tortugas marinas, cetáceos, delfines, peces, entre otros invertebrados y mamíferos marinos. El tráfico ilegal de especies marinas en Colombia es una problemática ambiental que afecta el desarrollo de ecosistemas marítimos y costeros, atentando contra la preservación de especies, el equilibrio de la fauna marina y la consagración del bienestar ecosistémico, lo cual demanda una gran atención e intervención por parte de las entidades protectoras y reguladoras de los ecosistemas marítimos y entre ellas se destaca la Armada Nacional, como agente de especial jurisdicción en zonas marítimas en el Pacífico y el Caribe, fluviales y áreas terrestres que se conectan directamente en la influencia de estas zonas.

El rol de la Armada Nacional en el tráfico ilegal de especies marítimas es crucial para evitar la degradación del ecosistema, ya que su ejercicio protector puede activar sistema de vigilancia que regule la acción humana y que disminuya los actos delictivos con dichas especies. En el presente manuscrito se identifica y analiza el rol de la Armada Nacional y su intervención en el tráfico marítimo desde el Ordenamiento Marino Costero, la jurisprudencia que regula el medio ambiente y la constitución de políticas, programas, proyectos y convenciones de diversos actores que han dispuesto un estatuto para la protección de especies marinas.

\section{Metodología}

Para el desarrollo del artículo se emplea el método de Revisión y Análisis Documental donde el procedimiento empleado es metódico, no busca una solución inmediata, sino que orienta Ediciones EFIM un proceso por un examen detenido y riguroso de documentos de naturaleza académica y normativa y de las conexiones que mantienen entre sí, a través de los siguientes pasos: 1) Rastrear documentos existentes y disponibles; para clasificar los documentos de acuerdo a su relación con la temática de estudio; 2) Selección de los documentos más pertinentes para los propósitos de la investigación; 3) lectura en profundidad del contenido de los documentos seleccionados, para extraer elementos de análisis y consignarlos en notas marginales, en donde se registraron los patrones, tendencias, convergencias y contradicciones que se iban descubriendo; 4) Finalmente se leyeron en forma cruzada y comparativa los documentos en cuestión, ya no sobre la totalidad del contenido de cada uno, sino sobre los hallazgos previamente realizados, a fin de construir una síntesis comprensiva total, sobre la problemática analizada (Quintana y Montgomery, 2006).

Teniendo en cuenta lo anterior, en el inciso "Resultados y discusión" se incluyen los siguientes ítems: 1) Rol de la Armada de la Republica de Colombia (ARC) en la protección del medio ambiente marino; 2) La Armada Nacional y el combate del tráfico ilegal de especies marinas; 3) La Armada Nacional y el ordenamiento marino costero en contra del tráfico ilegal de especies marinas. Los cuales permitirán identificar, abordar y analizar la problemática objeto de estudio.

\section{Resultados y discusión}

\section{Rol de la armada de la república de Colom- bia (ARC) en la protección del medio am- biente marino}

La Armada Nacional de Colombia ARC es la fuerza naval de las fuerzas militares de Colombia constituida por el cuerpo Naval y la Infantería de Marina. La ARC tiene como misión garantizar la integridad territorial, la protección de Estado y sus instituciones de la jurisdicción en las zonas marítimas en el Pacífico y el Caribe, zonas fluviales y algunos espacios terrestres:

"La protección de los recursos marinos renovables y no renovables, el combate al tráfico ilícito de especies, el control de la explotación 
irracional de recursos y la protección de diversas especies en peligro de extinción o sobre las cuales existen vedas, la contribución a las actividades costa afuera seguras y amigables con el medio marino son algunas de las acciones que la Institución desarrolla en forma permanente enmarcadas en un compromiso social con los colombianos". (Armada Nacional Republica de Colombia, 2014a, p.36)

"La Armada Nacional se encarga del ordenamiento territorial y la justicia ambiental en planteamientos específicos con objetivos enfocados en el desarrollo económico, equilibrio de las regiones, mejoramiento de la calidad de vida en las personas y la protección del medio ambiente como fundamento de empleo racional y territorial de los instrumentos necesarios del diseño, la formulación e implementación de políticas públicas en las instituciones jurídicas presentando un fuerte impacto en el sistema ambiental" (Armada Nacional Republica de Colombia, 2014a).

La Constitución Política (1991) impone la determinación de valores y bienes que atraigan las condiciones sociales para garantizar condiciones que permitan contar con un medio ambiente potente en recursos sanos y acciones humanas adecuadas (artículos 2, 4, 5 y 6), de esta forma la Armada Nacional se apropia de los valores constitucionales para proteger el medio ambiente que incluye los seres humanos, fauna, flora y objetos considerados patrimonio nacional.

El Estado debe asumir una mayor intervención como un respaldo constitucional donde se hace necesario un salto cuantitativo y cualitativo en la planificación de un ámbito supra local, por tanto los problemas ambientales reconocen un fuerte impacto en el sistema económico en el enfoque de los asentamientos humanos de escasos recursos, en posición de vulnerabilidad y/o grupos minoritarios, que la Armada Nacional protege desde su jurisprudencia.

\section{La armada nacional y el combate del tráfico ilegal de especies marinas}

El tráfico ilegal de especies marinas es una problemática grave que atenta contra biodiversidad del país, estableciendo una seria condición de amenaza y riesgo para muchas variedades de fauna y flora, que se suma a las dificultades originadas por la destrucción de ecosistemas, contaminación, desnutrición y fragmentación de habitas, la cacería ilegal y profanación de las especies que se comercializa a nivel nacional e internacional.

Según la Queensland Goverment (2002) “ $L a$ fragmentación del hábitat generalmente se considera una reducción en la continuidad de un hábitat a través de perturbaciones o pérdidas. Esta perturbación o pérdida puede ser el resultado de perturbaciones naturales o no naturales" (p.6), en el caso del tráfico ilegal de especies marinas se establecen actos delictivos ilegales que van en contra de la biodiversidad, la estabilidad y el bienestar ambiental y por ende deben ser procesados por las instituciones a cargo.

Así mismo, "Colombia es un país con vocación marítima por naturaleza, su valiosa posición geográfica entre dos océanos, le permite el acceso y conexión por el mar a los principales centros económicos del mundo" (Armada Nacional Republica de Colombia, 2014b, p.5), económicamente es una ubicación estratégica que permite la comercialización de sus productos, sin embargo también es una desventaja en cuanto al tráfico ilegal de especies marinas que se desarrolla en los mismos espacios geográficos.

En concordancia lo anterior, Castaño (2017) menciona que el tráfico ilegal de especies marinas y especies silvestres en general "Es considerado un problema de seguridad nacional que se da sobre todo en época de vacaciones y se mueve por rutas turísticas y zonas de tránsito" (p.2), puesto que solo en el 2017 se incautaron más de 246.320 especies silvestres que se dirigían a la comercialización, las cuales se encontraban en condiciones desfavorables y peligrosas, sin las condiciones mínimas de seguridad y transporte de las especies, estableciendo una ruta de supervivencia con pocas posibilidades de cruzar una ruta segura, considerado como maltrato, tortura y violación a la fauna y flora del país.

Ante esta problemática la Armada Nacional de Colombia en su ejercicio de proteger y vigilar los diversos y únicos recursos naturales combaAnfibios ISSN: 2665-1513 
te el tráfico ilegal de flora y fauna, con estrategias de vigilancia, control y operativos de inteligencia a lo largo y ancho del territorio nacional, especialmente en espacios marítimos, fluviales y las vías terrestres en coordinación con la Policía Nacional; "Entre agosto y octubre, elementos del Batallón 17 de la Brigada de Infantería de Marina No. 1 desarrollaron una operación sobre el río Magdalena, la principal arteria fluvial de Colombia" (Pelcastre, 2016, p.5). Ambas instituciones luchan contra el tráfico ilegal de vida silvestre y la tala y comercio ilegal de madera y solo en el 2017 han "Logrado la captura de 5.668 personas por la comisión de delitos contra el ambiente" (Castaño, 2017, p.2), fortaleciendo la seguridad y defensa del medio ambiente.

Finalmente, Pelcastre (2016) establece que la importancia de la acción de la Armada Nacional en el tráfico ilegal de especies marinas es fundamental para atacar la problemática con estrategias que vigilen, controlen y prevengan ejercicios delictivos que pongan en riesgo la biodiversidad de espacios marítimos a nivel nacional. Su rol aporta seguridad y defensa del medio ambiente, no solo de especies marinas sino de la fauna y flora a nivel general, por ende su ejercicio estratégico para atender la problemática debe centrarse en las zonas más frágiles y establecer relaciones de acompañamiento estratégico con otras entidades como la Policía Nacional, Gobiernos departamentales y regionales, al igual que campañas de protección y conservación con las comunidades.

\section{La armada nacional y el ordenamiento mari- no costero en contra del tráfico ilegal de es- pecies marinas}

La gran riqueza en recursos naturales de todo país está concentrado en las zonas marino costero al poseer las condiciones climáticas y ambientales para generar amplios productos y servicios de productividad y comunicación a nivel nacional e internacional, que alternadas con el turismo se convierten en un sistema altamente potencial, sin embargo el valor de esta riqueza más que un beneficio se convierte en una zona de empleo y explotación desmedida con distintos fines que causan caos y depresión en Ediciones EFIM el territorio, por tal razón desde el Programa de Naciones Unidas para el Medio Ambiente (PNUMA), UNESCO, ONGs y Foro Global sobre los Océanos, las Costas y las Islas (FGO) los gobiernos de cada país son convocados a crear y aplicar herramientas de ordenamiento y manejo costero con el objetivo de proteger y conservar en óptimas condiciones los mares, en especial en las zonas costeras mediando los conflictos por la competencia de estas zonas desde cada estado y plateando alternativas eficaces para solucionar los problemas de degradación de los recursos costeros, que en Colombia han sido apropiadas por la Armada Nacional (Naciones Unidas, 2003).

Dicho esto, el manejo costero integrado MCI en el territorio colombiano surge a partir a la Política Nacional Ambiental de Zonas Costeras de 1997 que tiene por objetivo restaurar los entornos y reservas naturales del sector costero del país y al mismo tiempo se busca mitigar toda acción que atente contra los bienes y servicios que provee este ecosistema, a través de un proyecto de formación para la población del territorio que requiere de prácticas de manejo y gestión de los recursos de su entorno (Minambiente, 2004).

Del mismo modo, la política MCI ha enfatizado en proyectos con impactos relevantes en amenazas a los ecosistemas marinos en los que se encuentra la planificación de sistemas que permitan contrarrestar los efectos del aumento del nivel del mar en Cartagena de Indias y Tumaco en el pacifico, igualmente se ha realizado una actualización y ajuste del diagnóstico y zonificación de los manglares de la zona costera que comprende desde el tajamar occidental de Bocas de Ceniza hasta Galerazamba. Además la Convención para la Conservación de los Recursos Vivos Antárticos (CCRVMA) pretende promover la explotación racional de los recursos marinos y así evitar rupturas y desequilibrios significativos en las zonas de gran valor natural previniendo la disminución de cualquier especie, en especial las que se encuentran en vía de extinción, de esta forma se minimizan los riesgos o cambios en el ecosistema marino y para ello se establecen límites y medidas educativas 
que regulen las actividades de la población para evitar la alteración de los ecosistemas.

Por su parte, El Convenio de las Naciones Unidas sobre el Derecho del Mar (CONVEMAR, 1999) regula el ordenamiento marino de la alta mar, a través del de estudios e investigaciones que demuestran los índices significativos de afección a este ecosistema y como ello contribuye a la degradación de todas y cada una de las especies que yacen en el paneta tierra y la Armada Nacional combate dichas problemáticas como programas de supervisión permanente que conlleve a la erradicación de actos delictivos que atenta contra las personas, la fauna y la flora del país.

Con base en lo descrito, la gestión de la Armada Nacional basada en la importancia del ecosistema ha sido una necesidad que surge a partir de los informes de la Comisión Pew para los Océanos (Pew Oceans Comission) que tiene por objetivo la regulación y coordinación de las acciones humanas que buscan el aprovechamiento desmedido de alimentos y recursos que ofrecen estas zonas, las cuales han contribuido al fenómeno de explotación, desequilibrio y contaminación (Armada Nacional Republica de Colombia, 2014b). Es por esto, que en primera pretensión evalúa el impacto que sufren los peces por la degradación de hábitats y se crea un mecanismo de regulación donde se delimitan las áreas aptas para la pesca, la recuperación de espacios, estudio de las características oceanográficas y valores de los ecosistemas, en segunda instancia fomentar las técnicas preventivas para evitar estos problemas a través de instrumentos voluntarios, como el Código de Conducta para la Pesca Responsable, grupos de apoyo de limpieza, reglamentos y espacios informativos para el turismos, entre otros.

\section{Biodiversidad marina y su explotación}

La globalización ha sumergido a todo el planeta en una experiencia de relación y transformación económica que integra medios de explotación, expansión, diversos avances tecnológicos y científicos en pro de un proceso de evolución sumamente veloz, en relación a los cambios que ha manifestado el territorio de acuerdo a la preservación de las tradiciones culturales, seguridad social, impacto ambiental y sostenibilidad. Las diversas problemáticas que aquejan el territorio marítimo costero colombiano se ven sumergidas en un conflicto político, económico y ambiental que degrada la calidad de vida del ecosistema.

De acuerdo con Ministerio de Ambiente y Desarrollo Sostenible (2014) "La biodiversidad reconocida como la "variabilidad de organismos vivos, incluidos, entre otros, los ecosistemas terrestres y marinos y otros ecosistemas acuáticos, y los complejos ecológicos de los que forman parte; comprende también la diversidad genética dentro de cada especie, entre las especies y los ecosistemas" (p.11), lo cual indica que la biodiversidad es un sistema explícito en el que cada especie cumple una función de demanda e interdependiente con los beneficios del contexto, donde la acción humana solo debe ser medidora y no un arma de destrucción que debilite el ecosistema, el medio ambiente y por ende su propia vida.

De otro lado, CEPAL (2001) sostiene que los ecosistemas y cada una de sus especies presentan diferentes expresiones de la vulnerabilidad social, que van desde la baja vigilancia, la contaminación, la explotación por caza en situaciones de desarrollo económico como práctica cultural, hasta la comercialización y tráfico de especies como ejercicio ilegal que busca primar el enriquecimiento ilícito por encima del equilibrio de la biodiversidad ecosistémica. El ejercicio de la Armada Nacional es fundamental para intervención de zonas marítimas y fluviales, es indispensable el establecimiento de áreas de protección y control que involucren a entidades y corporaciones ambientales, de esta forma se amplían los canales de prevención y vigilancia, en donde se debe capturar a toda persona que agreda de una forma u otra a las especies marinas desde el tráfico ilegal para la comercialización al interior y exterior del territorio colombiano.

De esta forma la Armada Nacional debe reducir y eliminar todo ejercicio de sobre explotación tráfico de especies marinas, ya que estas aportan Anfibios ISSN: 2665-1513 
al desarrollo ecosistémico marino indispensable para la preservación de los ciclos y procesos ecológicos, los cuales incluyen una unidad funcional compuesta por "Categorías de soporte, aprovisionamiento, regulación y cultura para el desarrollo de los diferentes sistemas culturales humanos en todas sus dimensiones (político, social, económico, tecnológico, simbólico, mítico $y$ religioso)" (Ministerio de Ambiente y Desarrollo Sostenible, 2014, p.11).

\section{Conclusiones}

Las afecciones de las actividades de pesca, el turismo y el saqueo legal o ilegal al ecosistema marino costero ha desencadenado un impacto nacional que reduce la calidad de vida y el tiempo de preservación de cada especie marina, por tanto es imprescindible acudir a mecanismos de regulación que involucre la participación de cada entidad que está implicada en la explotación y degradación de cada área, desde lo que compete a las funciones de la Armada Nacional en el cuerpo Naval y la Infantería de Marina, con el apoyo de instituciones reguladoras realizando operaciones de cooperación, asistencia humanitaria y apoyo.

Sin el ejercicio pertinente de la Armada Nacional el tráfico ilegal de especies puede masificarse y extenderse a lo largo y ancho del territorio, ya que esta organización da cobertura a los principales mares y puertos, por lo tanto es fundamental la implementación del Plan Estratégico Naval con esfuerzos especiales en zonas de gran vulneración, estableciendo en las mismas comunidades un plan de sensibilización y formación para que reconozcan la problemática del tráfico ilegal de especies marinas como una cuestión que repercute en sus familias y su ecosistema, al igual que ofrecer oportunidades de desarrollo económico en zonas fluviales, mares y puertos orientadas a otras actividades basados en el cuidado, la planificación, la preservación y la rentabilidad de la zona, todo ello con el apoyo del Gobierno Nacional y las entidades ambientales desde la responsabilidad social.

\section{Referencias bibliográficas}

Armada Nacional Republica de Colombia. (2014a). Políticas Institucionales. Bogotá, Colombia. Gobierno de Colombia.

Armada Nacional Republica de Colombia. (2014b). Plan Estratégico Naval. Bogotá, Colombia. Gobierno de Colombia.

Castaño, K. (2017). El tráfico ilegal de especies silvestres es una amenaza contra la biodiversidad. Bogotá, Colombia. Las2Orillas.

CEPAL. (2001). Las diferentes expresiones de la vulnerabilidad social en América Latina y el Caribe. Nueva York, Estados Unidos. Seminario Internacional Naciones Unidas y Centro Latinoamericano y Caribeño de Demografía CELADE.

Constitución Política Colombiana. (1991). Asamblea Nacional Constituyente. Bogotá, Colombia, 6 de Julio de 1991.

CONVEMAR. (1999). Convención de las Naciones Unidas sobre el Derecho del Mar. Nueva York, Estados Unidos. Naciones Unidas.

MINAMBIENTE. (2004). Política nacional ambiental para el desarrollo sostenible de los espacios oceánicos y las zonas costeras e insulares de Colombia. Bogotá, Colombia. Dirección General de Ecosistemas.

Ministerio de Ambiente y Desarrollo Sostenible. (2014). Términos de referencia para la elaboración del estudio de impacto ambiental explotación proyectos mineros. Bogotá, Colombia. MINAMBIENTE.

Naciones Unidas. (2003). Informe del Concejo de Administración. Bogotá, Colombia. Asamblea General.

Pelcastre, J. (2016). Armada Nacional de Colombia enfocada en la seguridad y defensa del medio ambiente. Bogotá, Colombia. Revista Digital Militar Diálogos. 
Mario Alberto Correo Segura, Diego Vela Ordoñez, Cristhian Mendoza Pérez

Queensland Goverment (2002). Fauna Sensitive Road Design: volume 1. Capitulo3. Nueva York, Estados Unidos. Department of Transport and Main Roads.

Quintana, A., \& Montgomery, W. (2006). Psicología: Topicos de actualidad. Lima: UNMSM. 